\title{
Listening to your heart and feeling yourself: effects of exposure to interoceptive signals during the ultimatum game
}

\author{
Bigna Lenggenhager $\cdot$ Ruben T. Azevedo • \\ Alessandra Mancini · Salvatore Maria Aglioti
}

Received: 6 April 2013 / Accepted: 5 July 2013 / Published online: 20 July 2013

(C) Springer-Verlag Berlin Heidelberg 2013

\begin{abstract}
The ultimatum game (UG) is commonly used to study the tension between financial self-interest and social equity motives. Here, we investigated whether experimental exposure to interoceptive signals influences participants' behavior in the UG. Participants were presented with various bodily sounds-i.e., their own heart, another person's heart, or the sound of footsteps-while acting both in the role of responder and proposer. We found that listening to one's own heart sound, compared to the other bodily sounds: (1) increased subjective feelings of unfairness, but not rejection behavior, in response to unfair offers and (2) increased the unfair offers while playing in the proposer role. These findings suggest that heightened feedback of one's own visceral processes may increase a selfcentered perspective and drive socioeconomic exchanges
\end{abstract}

Bigna Lenggenhager and Ruben T. Azevedo share first authorship.

B. Lenggenhager $\cdot$ R. T. Azevedo $\cdot$ S. M. Aglioti

IRCCS Fondazione Santa Lucia, Via Ardeatina 306,

00100 Rome, Italy

\section{Present Address:}

B. Lenggenhager $(\triangle)$

University Hospital of Child and Adolescent Psychiatry,

University of Bern, Bollingenstrasse 111, 3000 Bern 60,

Switzerland

e-mail: bigna.lenggenhager@gmail.com

R. T. Azevedo $\cdot$ A. Mancini $\cdot$ S. M. Aglioti

Department of Psychology, University of Rome "La Sapienza",

Via dei Marsi 78, 00185 Rome, Italy

Present Address:

A. Mancini

Institut des Sciences Cognitives, CNRS, 67 Boulevard Pinel,

69675 Bron, France accordingly. In addition, this study introduces a valuable procedure to manipulate online the access to interoceptive signals and for exploring the interplay between viscerosensory information and cognition.

Keywords Interoception · Heartbeat - Social cognition · Fairness · Economic game

\section{Introduction}

Social preferences such as altruism, reciprocity and fairness, are undeniably central to human interactions in daily life. However, by definition, choosing to behave altruistically may be costly at the personal level (Trivers 1971). Recently, psychology, neuroscience and behavioral economics started to investigate which brain regions, personality traits and context facilitate attempt to solve social dilemmas. A routinely employed task is the ultimatum game (UG) (Güth et al. 1982), where a proposer decides how to divide a sum of money, and a responder accepts or rejects the proposed division. If the offer is accepted, the proposal is implemented, and if rejected, both players receive nothing. Since the proposer has to offer more than 0 , from a strictly utilitarian point of view the responder should always accept. However, participants systematically reject unfair offers, preferring to gain nothing rather than accepting unequal distribution of resources (Camerer 2003). This form of pro-social punishment behavior is associated with a strong emotional reaction. In keeping with this finding, other studies have highlighted the crucial influence of specific emotional traits and states on the acceptance behavior in the UG. For example, experimentally induced sadness (Harle and Sanfey 2007), anger (Andrade and Ariely 2009) and disgust (Moretti and di 
Pellegrino 2010) resulted in higher rejection rates of unfair offers, thus confirming the link between emotional states and rejection behavior (Sanfey et al. 2003).

Importantly, mounting evidence suggests that emotion experience per se (independent of its content) is mediated by the access to internal bodily signals [i.e., interoception (Craig 2002; Sutterlin et al. 2011)]. At a neural level, attention toward bodily states, such as own heart rate, engages activity in the insula, ACC and somatosensory areas, i.e., a neural network strongly overlapping with the network involved in emotional experience (Zaki et al. 2012) and unfair-related decision making during the UG (Sanfey et al. 2003; Tabibnia et al. 2008). However, little is known about how interoceptive responses, and the awareness of them, influence emotional appraisal of unfair offers and modulate rejection behavior in the UG. Initial evidence derives from the finding of a linear relationship between changes in heart rate and rejection behavior (Osumi and Ohira 2009). Moreover, interoceptive accuracy has been found to moderate the relationship between changes in electrodermal activity to a given proposal and the behavioral rejection of the offer related to it (Dunn et al. 2012). Together, these results suggest an important link between the interoceptive system and the decision making task.

Here, we sought to investigate whether exposure to interoceptive signals influences participant's behavior in an UG. The influence of interoception has been shown on various cognitive or emotional tasks (e.g., attention, (Matthias et al. 2009), decision making (Werner et al. 2009), emotional intelligence (Schneider et al. 2005), empathy (Schneider et al. 2005) and tendency to general anxiety (Stewart et al. 2001). This has usually been assessed by grouping participants into good versus bad perceivers based on a heartbeat tracking task (Schandry and Weitkunat 1990) or heartbeat discrimination task (Critchley et al. 2004) and comparing the groups in a between-group design. Here, we used a within-participants approach and sought to manipulate the salience of interoceptive signals through experimental manipulation (compare Ainley et al. 2012) by exposing participants to three different bodily sounds, i.e., one's own heart, another person's heart or footstep sounds.

Capitalizing on a previous study (Mancini et al. 2011), we adapted a bilateral version of the UG task where participants alternatively acted as proposer or responder while hearing different sounds. We predicted that online exposure to one's own heartbeat would increase interoceptive awareness (Fenigstein and Carver 1978) and thus induce heightened appraisal of one's own emotional responses. Thus, we expected that when listening to their own heartbeat, participants would perceive the unfairness of offers more intensively and exhibit higher rejection rate. As a consequence, lower offers were expected when playing in the role of the proposer (van't Wout et al. 2010).

\section{Methods}

\section{Participants}

Thirty healthy participants ( 18 female; age range 19-39 years (mean $=25.7, \mathrm{SD}=5.0$ ) volunteered to participate in the study. They were informed that the money earned during the economic game corresponded to their actual payoff in the game and were accordingly reimbursed with an amount between 27.4 and 43 euro ( mean $=34.7, \mathrm{SD}=3.7$ ). Participants were naïve as to the purposes of the study and gave their written consent. The experimental protocol was approved by the local ethics committee at the Fondazione Santa Lucia, and the study was conducted in accordance with the ethical standards of the 1964 Declaration of Helsinki.

The ultimatum game

\section{General procedure}

Participants were required to play both the role of proposer and of recipient in a modified version of the UG. Before starting the game, they were told that they could see the faces of the other participants, located at two remote Italian Universities, by means of an online network. As in the classical version of the UG (Güth et al. 1982), the proposer's role was to decide how to split an amount of money (here always corresponding to 1 euro), while the recipient's role was to decide whether to accept or reject the proposed allocation. If the recipient rejects an offer, both players would receive nothing, while if he accepts it, each player would keep the allocated amount. To rule out the possibility of any negotiation between participants, subjects were assured that for each game, they would be randomly paired with an anonymous partner. Unbeknown to the participants, the game took place "against" a PC device, which was programmed using E-Prime software 1.2.

\section{Modification used in the present study}

In order to prevent participants from deciding on a priori strategy (e.g., always accept anything above 30 cents), they were not provided with explicit quantities of the different offers but with pictures showing the different quantities with two cents coins (see Fig. 1). Pretests in a different sample of $n=12$ healthy controls showed that when presented with a forced-choice task, participants were reliably able to cluster the different splits into the corresponding categories.

The interoceptive manipulation

During the UG, participants listened to three sound categories: (1) their own heart, (2) someone else's heart and (3) 


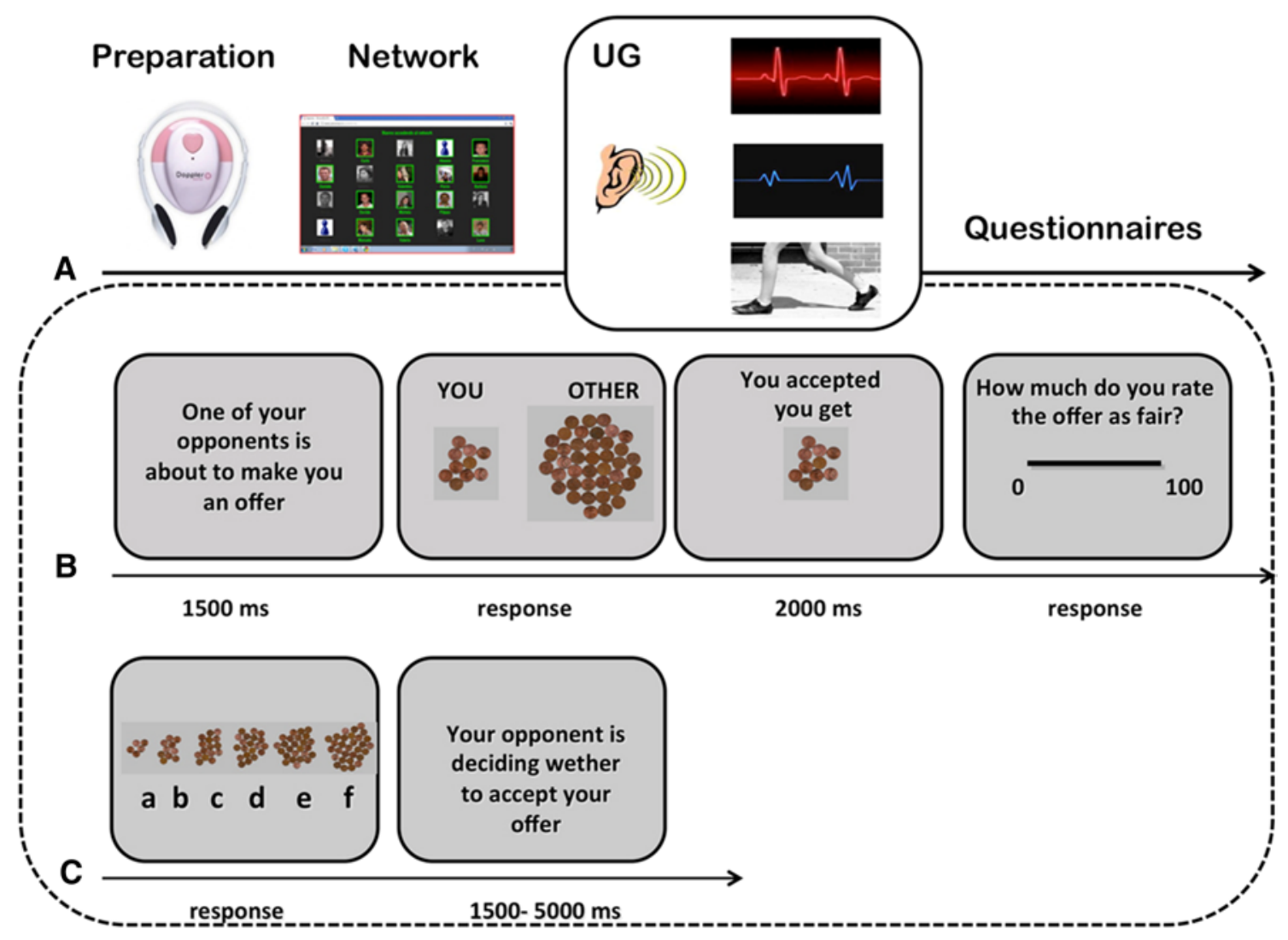

Fig. 1 Schematic procedure the experiment. a The experimental setup, $\mathbf{b}$ a trial as recipient and $\mathbf{c}$ a trial as a proposer

footsteps. Participants were told that a computer algorithm randomly assigned the sequence of the different sounds.

Own heartbeat For the own heartbeat condition, a fetal heart detector (Angle sound, Fetal Doppler, http://www. jumper-medical.com/) was attached with an elastic band over the participant's chest. The recorded sound was sent to sound processing software (Ableton Live 8.2.2, https:// www.ableton.com/), which reduced noise using low-pass frequency filters $(1.35 \mathrm{kHz})$ and equaled out volume differences. From there, it was played back to the participants' headphones.

Someone else's heartbeat The sound of another person's heart was previously recorded using the same method described above. Importantly, the mean heartbeat frequency (at rest) of the two conditions was matched as closely as possible (mean difference $=3.6$ beats $/ \mathrm{min}$; STE $=0.56$ ).

Footsteps Footstep sounds were matched to correspond to a mean frequency of a typical heartbeat $(1.17 \mathrm{~Hz})$. Furthermore, we used the Audacity software (version 1.2.6, http://audacity.sourceforge.net/) in order to make the sound slightly more irregular, making it less comparable to a natural fluctuation in the frequency of the heartbeat sound.

Experimental procedure

Subjects were seated in a comfortable armchair and were asked to relax their muscles but to stay alert. The headphones were mounted, and the Doppler device (angle sound) was placed over the participant's heart. A cover story was used to avoid participants gaining insight into the experiment's purpose, i.e., participants were told that sounds would be randomly assigned by the PC, and among all the possible sounds, there was the possibility to listen to the sound of their own heart, and therefore, a recording device should be prepared for this eventuality.

First, each participant was introduced to the rules of the UG and to the internet-based platform in order to familiarize participants with the procedure and to visualize the faces of the confederates. Then, the participants were told that the game would start.

Overall, each participant was tested in six experimental blocks: three in the role of recipient and three in the role 
of proposer. For each participant, the recipient block was repeated three times in random order for own heartbeat condition, other heartbeat condition and footsteps condition. On each block, participants completed 24 trials, for an overall amount of 144 iterations in the whole experiment. The order of the blocks was counterbalanced across participants.

Specific instructions presented on the screen prompted subjects to play the recipient or the proposer role (Fig. 1b). In the recipient blocks, participants received the following instructions (translated from Italian): "The computer randomly assigned you the role of responder. You may accept or reject the offers that come from your opponents. If you accept, the money will be divided according to the offer, and if you reject, neither of you will receive nothing." In the proposer blocks, participants received the following instructions (translated from Italian): "The computer randomly assigned you to the role of proposer. You may decide how to allocate the money. If your opponent accepts the offer, the money will be divided accordingly, and if he/she rejects the offer, no money will be given to any of you." Every recipient received four offers for each of six possible splits, that is, 90:10, 80:20, 70:30, 60:40, 50:50 and 40:60 eurocents. The recipient could accept or reject the offer by pressing a button (left to accept, right to reject) with their right hand. At the end of each interaction, a feedback lasting $4 \mathrm{~s}$ informed participants about how much each player received (again showing the picture of, e.g., 30 cents if accepted or, if the offer was rejected, an empty picture). In the proposer blocks, participants had to decide how to split money by choosing one of the six possible offers displayed on the screen (Fig. 1c). In these blocks, no feedback was provided to avoid any effect of the outcome on the subsequent offer.

At the end of the experiment, questionnaires were filled out, and the participants were debriefed about the purpose of the study. Overall, the experiment lasted about $1 \mathrm{~h}$.

\section{Subjective ratings of offers' fairness}

After each trial, participants were asked to assess the fairness of each offer on a visual analogue scale (VAS) ranging from 0 (unfair) to 100 (fair). The question (translated from Italian) was the following: "on a scale of $0-100$, where 0 corresponds to unfair and 100 to fair, how would you rate the offer you have just received?"

\section{Subjective ratings of attention to the game/sound}

After each block, participants evaluated how much attention they have paid to both the sound and the game (in randomized order) on a VAS scale ranging from 0 (no attention) to 100 (complete attention). This way, fluctuations of attention over blocks and conditions were assessed.
Manipulation check and phenomenological questions

At the end of the game, participants filled out a short questionnaire asking how they perceived the interaction during the game. In particular, a question on the recognition of their own heartbeat ("Do you think any of the sounds you heard was the sound of your own heart? If yes, in which block(s)?") was included.

Measures of interoceptive sensitivity and awareness

\section{Heartbeat counting (interoceptive sensitivity)}

Interoceptive sensitivity was measured using the classical "heartbeat tracking paradigm" (Schandry 1981). Participants were asked to focus on their heartbeat and internally count the number of heartbeats during 4 different intervals that were presented in random order: $25,35,45$ and $100 \mathrm{~s}$. Start and end of the counting period were signaled by an auditory cue. The real heartbeat was recorded using a finger oxymeter (Adinstruments). These two measures were used to calculate a sensitivity index (see "Ultimatum game").

\section{Recognition of one's own heart sound}

A total of 12 out of 30 participants correctly identified the sound of their own heart. The remaining 18 participants reported that they could not identify or misidentified the sound of their own heart. Unfortunately, we did not collect confidence levels related to this question that would allow us to have a quantitative measure of recognition. However, the final debriefing revealed that even the participants who correctly discriminated the sound of their own heart did so mostly on the basis of an "implicit feeling" guess.

\section{Data handling}

The fairness levels were clustered into 3 groups according to their fairness: unfair (10:90 and 20:80 offers), moderately unfair (30:70 and 40:60) and fair (50:50 and 60:40). A Kolmogorov-Smirnov test showed that most of the resulting variables of interest were not normally distributed, and thus, nonparametric statistics were used for acceptance rates, offers and fairness ratings. A Friedman test was performed to test the effect of condition (one's own HB, footsteps, other HB) on the three fairness levels. Moreover, Wilcoxon tests were used for post hoc analysis of significant effects of condition. The VAS scale on the attention was analyzed using parametrical measures, as the variables were normally distributed.

To split groups according to their interoceptive sensitivity, an index was calculated as the mean score of four heartbeat perception intervals $[25,35,45 \mathrm{~s}, 100 \mathrm{a}=$ according to 
the following transformation (see e.g., Schandry 1981; Tsakiris et al. 2011)]: $\frac{1}{4} \sum \frac{(1-\text { recorded heartbeats -counted heartbeats })}{\text { recorded heartbeats }}$ recorded (at the denominator). This transformation reveals a sensitivity index from 0 (i.e., low interoceptive sensitivity) to 1 (i.e., high interoceptive sensitivity). The median value of interoceptive sensitivity was 0.70 (s.d. 0.19). Using a median split method, the group of 27 participants (data of 3 participants were missing due to technical problems with the recording of the heartbeat) was split into two groups of high interoceptive sensitivity (good perceivers, mean heart beat perception 0.8 s.d. $0.08, n=14$ ) and low interoceptive sensitivity (bad perceivers, mean heartbeat perception 0.4 s.d. $0.23, n=13$ ).

\section{Results}

Ultimatum game

\section{Effect of the sounds on fairness judgment and acceptance rate}

When participants were playing as recipients, the effect of condition (own HB, footsteps, other HB) was analyzed for the fairness levels for acceptance rate and for fairness judgments. While no significant effect could be evidenced for the acceptance rate (all $p>0.05$, see Table 1a; Fig. 2a), the fairness judgments of low-fairness offers were significantly influenced by the presented sound (see Table 1b; Fig. 2b).

Post hoc analysis revealed that unfair offers were perceived as less fair while listening to one's own heartbeat as compared to when listening to another person's heartbeat or to footsteps (see Fig. 2b).

\section{Effects on offers made in the role of proposer}

When participants played in the role of proposer, there was again an effect of the experimental condition on the low-fairness offers only (see Table 1c). Post hoc analysis suggested that participants offered more unfair offers when listening to their own heart as compared to another person's heart or the footsteps (see Fig. 2c), which also resulted in a lower general sum of offers made (Friedman $7.5 p=0.023$ ).

Behavior of good versus bad perceivers

In order to test if the trait interoceptive sensitivity, as measured by the heartbeat counting paradigm, played a role in explaining the different influence of listening to sound categories on the UG, we tested whether the difference between conditions (e.g., own HB relative to footsteps) was modulated as a function of the sensitivity index by grouping the participants into good versus bad perceivers (see "Data handling"). Yet, neither of the significant effects (see Table 1) differed significantly between the two groups (all $p$ values $>0.05$ ).

Attention paid to the game in the different sound conditions

A $2 \times 3 \times 2$ ANOVA with the within-factors role (recipient, proposer), condition (one's own heartbeat, footsteps and other's heartbeat) and object (attention to game/ attention to sound) revealed a significant main effect of condition $\left(F_{(2,28)}=4.2, p=0.025,\right)$, as well as a main effect of object $\left(F_{(1,29)}=16.5, p<0.001\right)$ explained by a
Table 1 Effect of the different conditions on (a) acceptance rate and (b) the fairness judgment when playing as recipients. (c) Effect of the different conditions on the offers made when playing as proposer
Shown are the results of the Friedman test of the condition as well as the post hoc comparison (Wilcoxon). Significant effects are highlighted in bold

\begin{tabular}{llll}
\hline Fairness level & Chi square & $p$ value & Post hoc \\
\hline $\begin{array}{l}\text { (a) Acceptance rate } \\
\text { Low }\end{array}$ & 3.1 & 0.21 & \\
$\quad \begin{array}{l}\text { Middle } \\
\text { High }\end{array}$ & 0.9 & 0.63 & \\
$\begin{array}{l}\text { (b) Fairness judgment (VAS) } \\
\text { Low }\end{array}$ & 0.0 & 1 & \\
& $\mathbf{8 . 4}$ & $\mathbf{0 . 0 1 5}$ & $\begin{array}{l}\text { Own HB }>\text { footsteps }(\boldsymbol{p}=\mathbf{0 . 0 3}) \\
\text { Own HB }>\text { other HB }(\boldsymbol{p}=\mathbf{0 . 0 3})\end{array}$ \\
$\begin{array}{l}\text { Middle } \\
\text { High }\end{array}$ & & Other HB $=$ footsteps $(\mathrm{n} . \mathrm{s})$
\end{tabular}



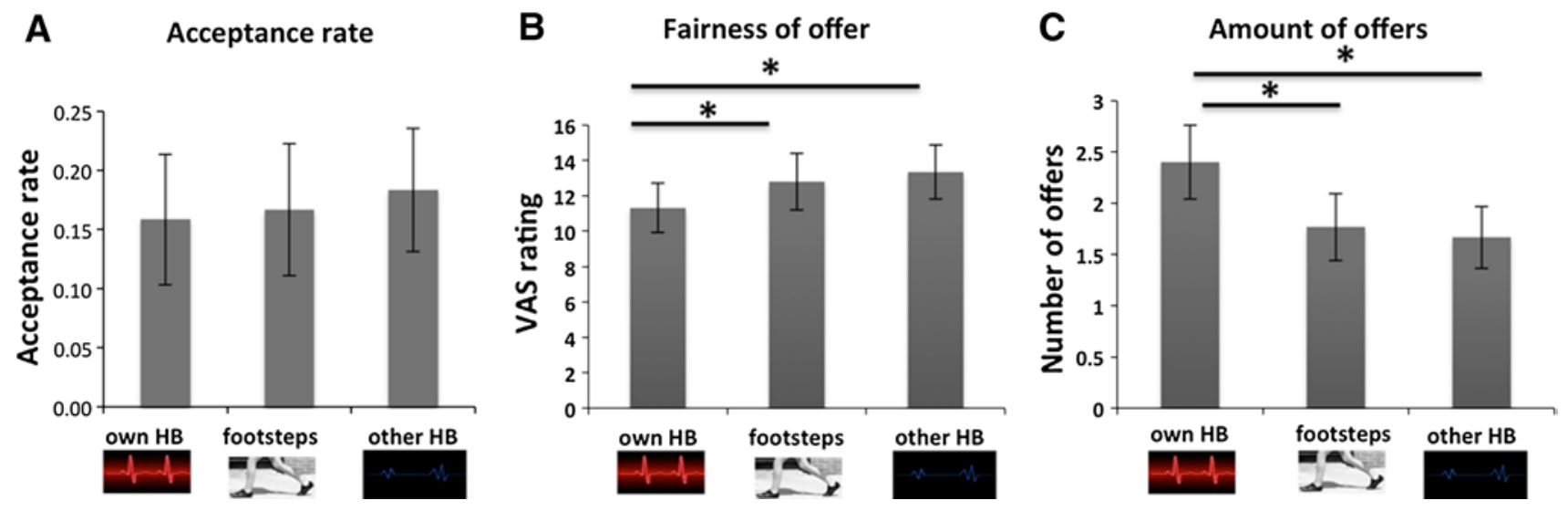

Fig. 2 a Mean value and standard error of acceptance rate to unfair offers (average response to 10:90 and 20:80 offers), b perceived fairness unfair offers (average response to 10:90 and 20:80 offers) as measured by the VAS scale during the three experimental conditions (mean and standard error). c Amount of unfair offers (sum of 10:90 and 20:80 offers) made when in the role of proposer (mean and standard error)

\section{Discussion}

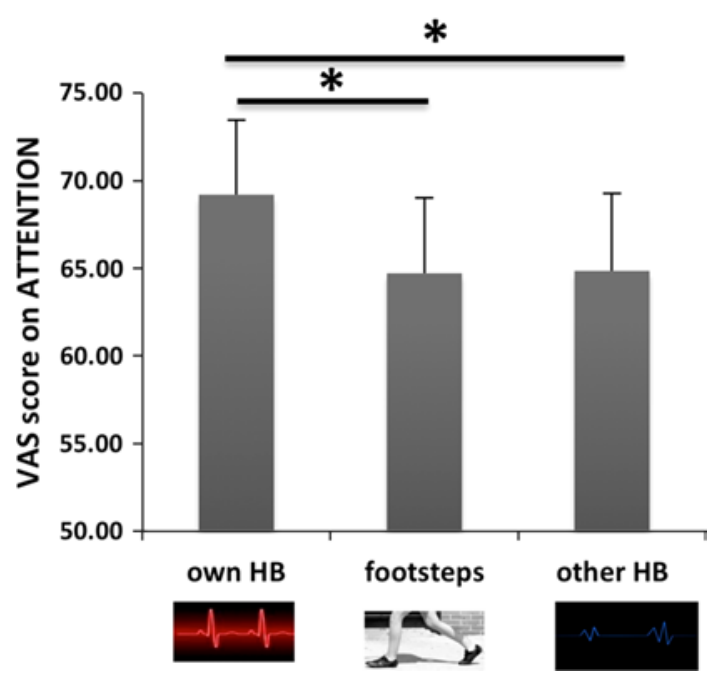

Fig. 3 Attention paid to the game during the different sound conditions. Depictured are mean values and standard errors

higher amount of attention being drawn to the game than to the sound and an interaction effect of role and object $\left(F_{(1,29)}=10.1, p=0.004\right)$. Post hoc $t$ tests of the main effect of condition suggest that participants were generally more attentive when hearing their own heartbeat as compared to the footsteps ( $t=2.4, p=0.02$, see Fig. 3) and a trend to more attentive as compared to other's heartbeat $(t=2.5, p=0.02)$. No significant difference between other's heartbeat and footstep was found $(t=0.6, p=0.95$, see Fig. 3). Post hoc $t$ tests of the interaction effect suggest that participants paid more attention to the game and less to the sound when they were in the role of the proposer as compared to when they were in the role of the recipient.
In the present study, we explored for the first time whether exposure to interoceptive signals during the UG may influence social economic decision making. We demonstrate that providing auditory online feedback of one's own heartbeat (1) increased subjective feelings of unfairness in response to unfair offers and (2) increased the unfair offers of participants playing in the proposer role. These findings provide compelling evidence for an influence of interoceptive information on decision making in socioeconomic exchange scenarios and contribute to the understanding of the emotional reactions to altruistic punishment in the context of the UG (Sanfey et al. 2003; Moretti and di Pellegrino 2010; Dunn et al. 2012). In addition, we suggest our approach introduces a new procedure for conveying interoceptive information to study the interplay between viscero-sensory information and cognition.

Influence of exposure to different bodily sounds on perceived fairness of offers

When acting in the role of recipient, we found that providing feedback about one's own cardiac processes enhanced the feeling of unfairness of very disadvantageous offers. Interestingly, such modulation was true only for the lowfairness offers, the type of offer frequently reported to have $50 \%$ chance of rejection (Nowak et al. 2000; Camerer 2003). We suggest that listening to their own heartbeat made participants attend more their reaction to receiving an unfair offer (e.g., anger, see (Pillutla and Murnighan 1996), leading to a change in the subjective appraisal of the monetary offers. 
Yet, enhanced feelings of unfairness were not accompanied by changes in acceptance behavior. A similar dissociation between appraisal and acceptance behavior was previously found in two repeated transcranial magnetic stimulation (rTMS) studies (Knoch et al. 2006; Baumgartner et al. 2011). Both studies found that the right dorsolateral prefrontal cortex to be specifically involved in rejection behavior but not in fairness judgment, suggesting distinct neural networks underpin these two processes. We thus suggest that despite the feelings of unfairness, participants might have been able to regulate punishment impulses enough and continued to act in a self-interested fashion, possibly using the expression of their enhanced inequity feelings as a less costly strategy to punishment (i.e., rejection). In keeping with this, it has been shown that recipients who are allowed to directly display their negative emotions to proposers exhibited fewer rejections with respect to those who were constrained to keep their emotions to themselves (Xiao and Houser 2005). Finally, recent research demonstrates that engagement in different cognitive strategies of emotional regulation impacts affective reactions to unfair offers and rejection (van't Wout et al. 2005; Grecucci et al. 2013b). The posterior insular cortex, an area that processes visceral interoceptive information (Craig 2009; Farb et al. 2013), was found to be particularly responsive to emotional modulation strategies (Grecucci et al. 2013b). In another study (Kirk et al. 2011), experienced Buddhist meditators, who may have higher interoceptive sensitivity, presented higher acceptance rates and preferentially activated the posterior insula during unfair offers, while controls recruited the anterior portion of the insular cortex, an area that has been shown to predict rejection behavior in the UG (Sanfey et al. 2003). The authors argued that meditators, likely attending internal body states, were better able to uncouple negative emotional responses from their behavior. Together with our results, these findings suggest that interoceptive signals may partly mediate cognitive appraisal of economic offers.

Influence of exposure to different bodily sounds on making offers

When acting in the role of the proposer, participants offered a lower total amount of money when listening to their own heart sound than when listening to the other sound categories. Interestingly, this effect was explained by the presence of a higher amount of unfair offers. This behavior does not seem strategic as in the classical UG participants usually expect their opponents to reject unfair offers (Frith and Singer 2008) and consequently offer an even or almost even split (Camerer 2003). Very few studies have investigated how emotional processing might affect behavior in the role of the proposer. Yet, two alternative explanations seem plausible to explain this finding. First, it has been shown that the amount of money proposed in the second interaction is related to the amount of money initially offered to the participant (e.g., van't Wout et al. 2010). This is in line with the appraisal theory, which suggests that specific cognitions are important antecedents of specific emotions and thereby specific action tendencies (e.g., Frijda et al. 1989; Smith and Ellsworth 1985) Thus, the cognitive appraisal of unfairness and the resulting emotion of anger when playing as a responder could have driven an enhancement of punitive behavior when playing as proposers. Second, as a more direct influence, interoceptive feedback might have enhanced a self-centered perspective taking. This idea is in line with data showing that participants with high interoceptive sensitivity are more resistant to bodily illusions and self-other confusions (Tsakiris et al. 2011), thus showing a stronger first person perspective. Furthermore presentation of the online heartbeat on a virtual body has been shown to change self-identification with it and self-location toward it, suggesting an important contribution of interoceptive signaling to center the self (Aspell et al. in press).

The influence of sounds is implicit and independent of interoceptive sensitivity

Listening to one's own heart as compared to another heart did influence judgment, behavior and attention during the game. In particular, we found increased attention to the game and to the sound during the own heart, which is in line with previous findings of an association between interoceptive awareness with increased divided attention (Matthias et al. 2009). Despite these significant influences, participants were, by and large, unable to consciously discriminate their own heart from that of another person as evidenced by post-experimental questionnaire. Moreovereven if this finding has to be taken with some caution due to a relatively small sample size-behavior or fairness appraisal in the UG does not seem to be explained by participants' scores in the heartbeat counting paradigm. This suggests that interoceptive sensitivity does not have a direct role in the observed modulation. It is thus likely that the (exteroceptive) exposure to interoceptive signals increased (implicit) interoceptive awareness even in participants who are not sensitive to changes in physiological activity. This is in line with recent literature that showed an influence of interoceptive signals on emotional, cognitive and even neurophysiological processes without awareness of it (e.g., Aspell et al. in press; Gray et al. 2007, 2010).

Interestingly, and in line with these findings, the only study that has previously explored the role of interoception on the UG did not find a direct relationship between interoceptive sensitivity as measured by the counting task and behavior (Dunn et al. 2012). Instead, the authors found 
that interoceptive accuracy moderated the relationship between electrodermal responses to unfair offers and rejection behavior, and only in participants classified as "good interoceptors." On the other hand, in "bad interoceptors," it was greater heart rate variability (and index of trait emotion regulation ability) that predicted reduced rejection rates (see also (Sutterlin et al. 2011)). The study thus demonstrates how individual differences in perceiving bodily signals interact with different strategies of emotional responding. While the measurement of individual differences in interoceptive sensitivity has proven to be a useful tool in the study of how this ability correlates with behavior or personality traits (Stewart et al. 2001; Herbert et al. 2007; Matthias et al. 2009; Pollatos et al. 2009; Werner et al. 2009; Dunn et al. 2012), this approach may not always provide the most complete description of the role of interoception in cognition. In fact, several studies found the effects of cardiac interoceptive accuracy to be context dependent or to mediate/or be mediated by other variables (Bogaerts et al. 2005, 2008; Pollatos et al. 2009; Dunn et al. 2012).

In addition, several concerns have been raised about the standard measurements (Jones 1994; Knapp-Kline and Kline 2005; Khalsa et al. 2009; Ceunen et al. 2013), as such approaches rely on between participant designs making them more prone to confounds of other related personality traits. In some cases, manipulating attention to interoceptive signals within participants may constitute a more flexible and comprehensive approach to study the interplay between visceral states, cognition and social behavior. Here, we introduce a novel procedure to manipulate access to interoceptive signals in an event-related and fairly implicit way.

Finally, it is worth noting that presenting feedback of one's own heart sound is substantially different from providing feedback of heart rate by means of an auditory tone or light flash at the occurrence of a heartbeat (Gray et al. 2007). The sound of the heart, as translated by means of a Doppler device, conveys information such as systolicdiastolic interval, duration and changes in intensity of cardiac tones that better represent the cardiovascular activity, and thus is likely to provide a more comprehensive feedback on cardiac responses. Future research using this approach should be complemented with further measures such as indices of autonomic activity such as electrodermal activity and heart rate (Osumi and Ohira 2009; Dunn et al. 2012), brain activity (Sanfey et al. 2003; Baumgartner et al. 2011; Grecucci et al. 2013b) or trait and state anxiety (Paulus and Stein 2006; Hartley and Phelps 2012; Grecucci et al. 2013a) to help disentangle the dynamics associated with the feedback of own heart sound. We foresee that this manipulation might reveal itself as a valuable tool for the study of emotion regulation processes and the interplay between viscero-sensory information and emotional states.
Acknowledgments We thank Jane Aspell for proofreading. This study was supported by EU Information and Communication Technologies Grant (VERE project, FP7-ICT-2009-5, Prot. Num. 257695), the Italian Ministry of Health (and RF-2010-2312912). BL was supported by the Swiss National Science Foundation (grant PBELP3133268). RTA was supported by Fundação para a Ciência e a Tecnologia (FCT) and POPH/FSE (doctoral grant SFRH/BD/60517/2009).

\section{References}

Ainley V, Tajadura-Jimenez A, Fotopoulou A, Tsakiris M (2012) Looking into myself: changes in interoceptive sensitivity during mirror self-observation. Psychophysiology 49:1504-1508

Andrade EB, Ariely D (2009) The enduring impact of transient emotions on decision making. Organ Behav Hum Decis Process 109:1-8

Aspell JE, Heydrich L, Marillier G, Lavanchy T, Herbelin B, Blanke $\mathrm{O}$ (in press) Turning body and self inside-out: visualized heartbeats alter bodily self-consciousness and tactile perception. Psychol Sci

Baumgartner T, Knoch D, Hotz P, Eisenegger C, Fehr E (2011) Dorsolateral and ventromedial prefrontal cortex orchestrate normative choice. Nat Neurosci 14:1468-1474

Bogaerts K, Notebaert K, Van Diest I, Devriese S, De Peuter S, Van den Bergh O (2005) Accuracy of respiratory symptom perception in different affective contexts. J Psychosom Res 58:537-543

Bogaerts K, Millen A, Li W et al (2008) High symptom reporters are less interoceptively accurate in a symptom-related context. J Psychosom Res 65:417-424

Camerer BF (2003) Behavioural game theory. Princeton University Press, Princeton

Ceunen E, Van Diest I, Vlaeyen JW (2013) Accuracy and awareness of perception: related, yet distinct (commentary on). Biol Psychol 92:426-427

Craig AD (2002) How do you feel? interoception: the sense of the physiological condition of the body. Nat Rev Neurosci 3: 655-666

Craig AD (2009) How do you feel-now? the anterior insula and human awareness. Nat Rev Neurosci 10:59-70

Critchley HD, Wiens S, Rotshtein P, Ohman A, Dolan RJ (2004) Neural systems supporting interoceptive awareness. Nat Neurosci 7:189-195

Dunn BD, Evans D, Makarova D, White J, Clark L (2012) Gut feelings and the reaction to perceived inequity: the interplay between bodily responses, regulation, and perception shapes the rejection of unfair offers on the ultimatum game. Cognit Affect Behav Neurosci 12:419-429

Farb NA, Segal ZV, Anderson AK (2013) Attentional modulation of primary interoceptive and exteroceptive cortices. Cereb Cortex 23:114-126

Fenigstein A, Carver CS (1978) Self-focusing effects of heartbeat feedback. J Pers Soc Psychol 36:1241-1250

Frijda NH, Kuipers P, Ter Schure E (1989) Relations among emotion, appraisal, and emotional action readiness. J Pers Soc Psychol 57:212-228

Frith CD, Singer T (2008) The role of social cognition in decision making. Philosophical transactions of the Royal Society of London. Ser B Biol sci 363:3875-3886

Gray MA, Harrison NA, Wiens S, Critchley HD (2007) Modulation of emotional appraisal by false physiological feedback during fMRI. PLoS One 2:e546

Gray MA, Minati L, Paoletti G, Critchley HD (2010) Baroreceptor activation attenuates attentional effects on pain-evoked potentials. Pain 151:853-861 
Grecucci A, Giorgetta C, Brambilla P et al (2013a) Anxious ultimatums: how anxiety disorders affect socioeconomic behaviour. Cogn Emot 27:230-244

Grecucci A, Giorgetta C, Van't Wout M, Bonini N, Sanfey AG (2013b) Reappraising the ultimatum: an FMRI study of emotion regulation and decision making. Cereb Cortex 23:399-410. doi:10.1093/cercor/bhs028

Güth W, Schmittberger R, Schwarze B (1982) An experimental analysis of ultimatum bargaining. J Econ Behav Organ 3:367-388

Harle KM, Sanfey AG (2007) Incidental sadness biases social economic decisions in the ultimatum Game. Emotion 7: 876-881

Hartley CA, Phelps EA (2012) Anxiety and decision-making. Biol Psychiatry 72:113-118

Herbert BM, Pollatos O, Schandry R (2007) Interoceptive sensitivity and emotion processing: an EEG study. Int J Psychophysiol Off J Int Organ Psychophysiol 65:214-227

Jones GE (1994) Perception of visceral sensations: a review of recent findings, methodologies, and future directions. Jessica Kingsley Publishers, London

Khalsa SS, Rudrauf D, Sandesara C, Olshansky B, Tranel D (2009) Bolus isoproterenol infusions provide a reliable method for assessing interoceptive awareness. Int J Psychophysiol Off J Int Organ Psychophysiol 72:34-45

Kirk U, Downar J, Montague PR (2011) Interoception drives increased rational decision-making in meditators playing the ultimatum game. Front Neurosci 5:49

Knapp-Kline K, Kline JP (2005) Heart rate, heart rate variability, and heartbeat detection with the method of constant stimuli: slow and steady wins the race. Biol Psychol 69:387-396

Knoch D, Pascual-Leone A, Meyer K, Treyer V, Fehr E (2006) Diminishing reciprocal fairness by disrupting the right prefrontal cortex. Science 314:829-832. doi:10.1126/science.1129156

Mancini A, Betti V, Panasiti MS, Pavone EF, Aglioti SM (2011) Suffering makes you egoist: acute pain increases acceptance rates and reduces fairness during a bilateral ultimatum game. PLoS One 6:e26008

Matthias E, Schandry R, Duschek S, Pollatos O (2009) On the relationship between interoceptive awareness and the attentional processing of visual stimuli. Int J Psychophysiol 72:154-159

Moretti L, di Pellegrino G (2010) Disgust selectively modulates reciprocal fairness in economic interactions. Emotion 10:169-180

Nowak MA, Page KM, Sigmund K (2000) Fairness versus reason in the ultimatum game. Science 289:1773-1775

Osumi T, Ohira H (2009) Cardiac responses predict decisions: an investigation of the relation between orienting response and decisions in the ultimatum game. Int J Psychophysiol Off J Int Organ Psychophysiol 74:74-79

Paulus MP, Stein MB (2006) An insular view of anxiety. Biol Psychiatry $60: 383-387$
Pillutla MM, Murnighan JK (1996) Unfairness, anger, and spite: emotional rejections of ultimatum offers. Organ Behav Hum Decis Process 68:208-224

Pollatos O, Traut-Mattausch E, Schandry R (2009) Differential effects of anxiety and depression on interoceptive accuracy. Depress Anxiety $26: 167-173$

Sanfey AG, Rilling JK, Aronson JA, Nystrom LE, Cohen JD (2003) The neural basis of economic decision-making in the ultimatum game. Science 300:1755-1758

Schandry R (1981) Heartbeat perception and emotional experience. Psychophysiology 18:483-488

Schandry R, Weitkunat R (1990) Enhancement of heartbeat-related brain potentials through cardiac awareness training. Int $\mathrm{J}$ Neurosci 53:243-253

Schneider RS, Lyons JB, Williams M (2005) Emotional intelligence and autonomic self-perception: emotional abilities are related to visceral acuity. Pers Individ Differ 39:853-861

Smith CA, Ellsworth PC (1985) Patterns of cognitive appraisal in emotion. J Pers Soc Psychol 48:813-838

Stewart SH, Buffett-Jerrott SE, Kokaram R (2001) Heart beat awareness and heart rate reactivity in anxiety sensitivity: a further investigation. J Anxiety Disord 15:535-553

Sutterlin S, Herbert C, Schmitt M, Kubler A, Vogele C (2011) Overcoming selfishness: reciprocity, inhibition, and cardiac-autonomic control in the ultimatum game. Front Psychol 2:173

Tabibnia G, Satpute AB, Lieberman MD (2008) The sunny side of fairness: preference for fairness activates reward circuitry (and disregarding unfairness activates self-control circuitry). Psychol Sci 19:339-347

Trivers RL (1971) The evolution of reciprocal altruism. Q Rev Biol 46:35-57

Tsakiris M, Tajadura-Jimenez A, Costantini M (2011) Just a heartbeat away from one's body: interoceptive sensitivity predicts malleability of body-representations. Proceedings. Biol Sci Royal Soc 278:2470-2476

van't Wout M, Kahn RS, Sanfey AG, Aleman A (2005) Repetitive transcranial magnetic stimulation over the right dorsolateral prefrontal cortex affects strategic decision-making. NeuroReport 16:1849-1852

van't Wout M, Chang LJ, Sanfey AG (2010) The influence of emotion regulation on social interactive decision-making. Emotion 10:815-821

Werner NS, Jung K, Duschek S, Schandry R (2009) Enhanced cardiac perception is associated with benefits in decision-making. Psychophysiology 46:1123-1129

Xiao E, Houser D (2005) Emotion expression in human punishment behavior. Proc Natl Acad Sci USA 102:7398-7401

Zaki J, Davis JI, Ochsner KN (2012) Overlapping activity in anterior insula during interoception and emotional experience. NeuroImage 62:493-499 